\title{
The determination of minimum inhibitory concentrations of selected antimicrobials for porcine Haemophilus parasuis isolates from the Czech Republic
}

\author{
Kateřina Nedbalcová1, Monika Zouharová1, Daniel Šperling ${ }^{2}$ \\ ${ }^{1}$ Veterinary Research Institute, Brno, Czech Republic \\ ${ }^{2}$ Ceva Santé Animale S.A., Libourne, France \\ Received February 7, 2017 \\ Accepted May 31, 2017
}

\begin{abstract}
Haemophilus parasuis isolates obtained from pigs in the Czech Republic were tested for their susceptibility to amoxicillin, penicillin, ceftiofur, enrofloxacin, tetracycline, and tulathromycin by determination of minimum inhibitory concentrations using the broth microdilution method. The $H$. parasuis isolates were mostly susceptible to majority of tested antimicrobials (amoxicillin $90 \%$, penicillin $73.3 \%$, enrofloxacin $83.3 \%$, and tulathromycin $83.3 \%$ ). All isolates were susceptible to ceftiofur. On the other hand, no isolate was susceptible to tetracycline, $30 \%$ of tested isolates were intermediately susceptible, and $70 \%$ were resistant. These findings indicate that tested antimicrobials with the exception of tetracycline should be the preferred option used for the treatment of infection caused by $H$. parasuis but due to the potential transmission of resistance from animals to humans, the use of ceftiofur is considered as a last resort option in antimicrobial treatment of animals.
\end{abstract}

Bacterial infection, susceptibility, resistance, pigs

Haemophilus parasuis may be the cause of great economic loss for breeders due to the cost of antibiotic therapy and piglet death in the acute forms of the disease (Oliveira et al. 2001). In conventional herds, $H$. parasuis is predominantly involved in the respiratory syndrome but can also cause acute septicaemia or Glässer's disease, systemic illness characterized by fibrinous polyserositis, polyarthritis, and meningitis (Amano et al. 1994). Infections caused by $H$. parasuis can be enzootic and may be acute or chronic, depending on the immunological situation of the breeding herd. If $H$. parasuis entered into farms with immunologically naive animals, it can cause serious acute illness and the main affected age categories are early weaned piglets about the age of 4-6 weeks (Nicolet 1992). A high antigenic heterogeneity exists among $H$. parasuis strains. According to the currently worldwide accepted classification, 15 serovars of $H$. parasuis $(1-15)$ have been defined. However, it is necessary to say that a large number of non-typeable $H$. parasuis isolates also exist (Kielstein and Rapp-Gabrielson 1992).

Antibiotic treatment is one of the commonly used measures for the control of $H$. parasuis infections. However, the use of antimicrobial agents may lead to both selection and increase of resistance (Schwarz et al. 2001). Correct use of antimicrobial agents for treatment of bacterial infections requires the knowledge of the susceptibility of the infecting strain to antimicrobial agents to enhance efficacy and to prevent the emergence of resistance among other organisms, which may be causing respiratory diseases in pigs (De la Fuente et al. 2007). Moreover, continuous monitoring of the prevalence of drug resistance of bacterial pathogens to antimicrobials provides essential data on the state and development of the susceptibility in pathogens causing bacterial infections. These data are necessary for the prediction of an antimicrobial's efficacy in the initial treatment and to determine the position of individual substances as 'drugs of choice' or as alternative drugs in the therapy of infections. Susceptibility tests are therefore the basis of the formulation of objective antimicrobial treatment and prevention strategies (Nedbalcova et al. 2013).

Address for correspondence:

Dr. Kateřina Nedbalcová

Veterinary Research Institute

Hudcova 70, 62132 Brno, Czech Republic
Phone: 420533331217

Fax: 420541211229

E-mail: nedbalcova@vri.cz

http://actavet.vfu.cz/ 
The objective of this study was to determine the minimum inhibitory concentrations (MICs) for selected antimicrobials (amoxicillin, penicillin, ceftiofur, enrofloxacin, tetracycline, and tulathromycin) in the Czech field strains of Haemophilus parasuis according to the latest guidelines of the Clinical and Laboratory Standards Institute (CLSI 2013a; CLSI 2013b).

\section{Isolates}

\section{Materials and Methods}

Thirty isolates of $H$. parasuis (1-30) were obtained from diseased pigs from pig herds in the Czech Republic from 2014 to 2015. Haemophilus parasuis isolates were isolated on Columbia blood agar (OXOID, England) plates with 5\% sheep blood with a nurse strain of Staphylococcus aureus and then on chocolate blood agar (OXOID, England) plates with 5\% sheep blood for $\beta$-nikotinamid adenin dinucleotide growth dependence of $H$. parasuis for $24-48 \mathrm{~h}$ at $37{ }^{\circ} \mathrm{C}$, were confirmed by polymerase chain reaction test (Oliveira et al. 2001), and serotyped by modified co-agglutination test originally described for Actinobacillus pleuropneumoniae (Mittal et al. 1983). All isolates were stored at $-80^{\circ} \mathrm{C}$ in vials containing $0.25 \mathrm{ml}$ Foetal Bovine Serum Gold (PAA Laboratories GmbH, Austria) and $0.25 \mathrm{ml}$ of Cation Adjusted Mueller Hinton Broth II (CAMHB) (Becton, Dickinson and Company, USA). All isolates originated from animals without previous antimicrobial therapy during the last 3 weeks and only individual isolates from individual herds were included in this study.

MIC determination

The MICs of selected antimicrobials for the isolates of $H$. parasuis were determined by the standardized dilution micro-method for Actinobacillus pleuropneumoniae and Histophilus somni (CLSI 2013a; CLSI 2013b).

Preparation of microdilution trays

According to these documents, special microdilution trays were prepared. These trays included dilutions of tested antimicrobial agents (mg/l) in Veterinary Fastidious Medium (VFM) prepared according to CLSI (2013a). The tested antimicrobials and their concentrations are shown in Table 1.

Table 1. Microplate tray with concentrations of tested antimicrobials (mg/l).

\begin{tabular}{llllllllllll}
\hline PNC & AMX & EFT & TUL & TET & ENR & PNC & AMX & EFT & TUL & TET & ENR \\
\hline 8 & 4 & 16 & 64 & 64 & 4 & 8 & 4 & 16 & 64 & 64 & PC \\
4 & 2 & 8 & 32 & 32 & 2 & 4 & 2 & 8 & 32 & 32 & 2 \\
2 & 1 & 4 & 16 & 16 & 1 & 2 & 1 & 4 & 16 & 16 & 1 \\
1 & 0.5 & 2 & 8 & 8 & 0.5 & 1 & 0.5 & 2 & 8 & 8 & 0.5 \\
0.5 & 0.25 & 1 & 4 & 4 & 0.25 & 0.5 & 0.25 & 1 & 4 & 4 & 0.25 \\
0.25 & 0.125 & 0.5 & 2 & 2 & 0.125 & 0.25 & 0.125 & 0.5 & 2 & 2 & 0.125 \\
0.125 & 0.06 & 0.25 & 1 & 1 & 0.06 & 0.125 & 0.06 & 0.25 & 1 & 1 & 0.06 \\
0.06 & 0.03 & 0.125 & 0.5 & 0.5 & 0.03 & 0.06 & 0.03 & 0.125 & 0.5 & 0.5 & 0.03 \\
\hline
\end{tabular}

PNC - penicillin; AMX - amoxicillin; EFT - ceftiofur; TUL - tulathromycine; TET - tetracycline; ENR enrofloxacin; PC - positive growth control.

Inoculum preparation, inoculation and incubation of trays

The culture of $H$. parasuis grown on blood agar was re-suspended in $5 \mathrm{ml} \mathrm{CAMHB} \mathrm{(Becton,} \mathrm{Dickinson} \mathrm{and}$ Company, USA) and the density of the suspension was adjusted to a turbidity equivalent to that of a $0.5 \mathrm{McFarland}$ standard and $5 \mu \mathrm{l}$ of inoculum with the density of $0.5 \mathrm{McFarland}$ standard were transferred into all of the wells of the micro-titre plates containing $100 \mathrm{ml}$ of medium. The trays were incubated at $35^{\circ} \mathrm{C}_{\text {in }} \mathrm{CO}_{2}$ for $24 \mathrm{~h}$.

\section{Interpretation of results}

The MICs values were read at the lowest concentration of an antimicrobial agent that inhibited the visible bacterial growth in the wells. The break-points of tested antimicrobials for $H$. parasuis are derived from breakpoints for A. pleuropneumoniae and Pasteurella multocida according to CLSI (2013b) and they are shown in Table 2 .

\section{Quality control}

Quality control of the results was performed with reference strains of Actinobacillus pleuropneumoniae (ATCC 27090). The acceptable quality control ranges for tested antimicrobials are in CLSI document Vet 01-S2 (2013b). 
Table 2. The break-points of tested antimicrobials for Haemophilus parasuis.

\begin{tabular}{llll}
\hline & $\begin{array}{c}\mathrm{S} \\
\mathrm{mg} / \mathrm{l}\end{array}$ & $\begin{array}{c}\mathrm{I} \\
\mathrm{mg} / \mathrm{l}\end{array}$ & $\begin{array}{c}\mathrm{R} \\
\mathrm{mg} / \mathrm{l}\end{array}$ \\
\hline Penicillin & $\leq 0.25$ & 0.5 & $\geq 1$ \\
Amoxicillin & $\leq 0.5$ & 1 & $\geq 2$ \\
Ceftiofur & $\leq 2$ & 4 & $\geq 8$ \\
Tulathromycin & $\leq 16$ & 32 & $\geq 64$ \\
Tetracycline & $\leq 0.5$ & 1 & $\geq 2$ \\
Enrofloxacin & $\leq 0.25$ & 0.5 & $\geq 1$ \\
\hline
\end{tabular}

$\overline{\mathrm{S} \text { - susceptible; I - intermediately susceptible; } \mathrm{R} \text { - resistant }}$

\section{Results}

Distribution of MICs for penicillin, amoxicillin, ceftiofur, tulathromycin, tetracycline, and enrofloxacin in $H$. parasuis isolates, calculation of the $\mathrm{MIC}_{50}$ and $\mathrm{MIC}_{90}$ values and counts and percentages of susceptible, intermediately susceptible and resistant $H$. parasuis isolates to tested antimicrobials are in Table 3. As detected by the CLSI (2013a; 2013b)

Table 3. Interpretation of results

\begin{tabular}{|c|c|c|c|c|c|c|c|}
\hline & $\begin{array}{c}\text { Concentration } \\
\text { of antimicrobials }\end{array}$ & $\begin{array}{l}\text { Isolates } \\
\mathrm{N}(\%)\end{array}$ & $\begin{array}{c}\mathrm{C} \\
\mathrm{N}(\%)\end{array}$ & $\begin{array}{c}\mathrm{I} \\
\mathrm{N}(\%)\end{array}$ & $\begin{array}{c}R \\
N(\%)\end{array}$ & $\begin{array}{l}\mathrm{MIC}_{50} \\
(\mathrm{mg} / \mathrm{l})\end{array}$ & $\begin{array}{l}\mathrm{MIC}_{90} \\
(\mathrm{mg} / \mathrm{l})\end{array}$ \\
\hline \multirow[t]{7}{*}{ Penicilin } & $\leq 0.06$ & $6(20)$ & $22(73.3)$ & $2(6.7)$ & $6(20)$ & 0.25 & 4 \\
\hline & 0.125 & $3(10)$ & & & & & \\
\hline & 0.25 & $13(43.3)$ & & & & & \\
\hline & 0.5 & $2(6.7)$ & & & & & \\
\hline & 2 & $2(6.7)$ & & & & & \\
\hline & 4 & $1(3.3)$ & & & & & \\
\hline & 8 & $3(10)$ & & & & & \\
\hline \multirow[t]{5}{*}{ Amoxicilin } & $\leq 0.03$ & $10(33.3)$ & $27(90)$ & 0 & $3(10)$ & 0.06 & 0.25 \\
\hline & 0.06 & $12(40)$ & & & & & \\
\hline & 0.125 & $2(6.7)$ & & & & & \\
\hline & 0.25 & $3(10)$ & & & & & \\
\hline & 4 & $3(10)$ & & & & & \\
\hline \multirow[t]{4}{*}{ Ceftiofur } & $\leq 0.125$ & $16(53.3)$ & $30(100)$ & 0 & 0 & $\leq 0.125$ & 0.5 \\
\hline & 0.25 & $5(16.7)$ & & & & & \\
\hline & 0.5 & $6(20)$ & & & & & \\
\hline & 1 & $3(10)$ & & & & & \\
\hline \multirow[t]{5}{*}{ Tetracycline } & 1 & $9(30)$ & 0 & $9(30)$ & $21(70)$ & 2 & 64 \\
\hline & 2 & $15(50)$ & & & & & \\
\hline & 4 & $1(3.3)$ & & & & & \\
\hline & 32 & $1(3.3)$ & & & & & \\
\hline & 64 & $4(13.3)$ & & & & & \\
\hline \multirow[t]{6}{*}{ Enrofloxacin } & $\leq 0.03$ & $17(56.7)$ & $25(83.3)$ & 0 & $5(16.7)$ & $\leq 0.03$ & 1 \\
\hline & 0.06 & $2(6.7)$ & & & & & \\
\hline & 0.125 & $4(13.3)$ & & & & & \\
\hline & 0.25 & $2(6.7)$ & & & & & \\
\hline & 1 & $3(10)$ & & & & & \\
\hline & 2 & $2(6.7)$ & & & & & \\
\hline \multirow[t]{8}{*}{ Tulathromycin } & $\leq 0.5$ & $7(23.3)$ & $25(83.3)$ & $1(3.3)$ & $4(13.3)$ & 2 & 64 \\
\hline & 1 & $7(23.3)$ & & & & & \\
\hline & 2 & $4(13.3)$ & & & & & \\
\hline & 4 & $3(10)$ & & & & & \\
\hline & 8 & $2(6.7)$ & & & & & \\
\hline & 16 & $2(6.7)$ & & & & & \\
\hline & 32 & $1(3.3)$ & & & & & \\
\hline & 64 & $4(13.3)$ & & & & & \\
\hline
\end{tabular}

C - susceptibility; I - intermediate susceptibility; R - resistance; MIC - minimum inhibitory concentration $\mathrm{MIC}_{50}$ and $\mathrm{MIC}_{90}$ presented the lowest concentration of antimicrobial substances in $\mathrm{mg} / \mathrm{l}$ that inhibited the growth of $50 \%$ and $90 \%$ of isolates were determined by cumulative conversion (Schwarz 2010). 
methods for MIC determination the majority of the tested isolates were susceptible to the tested antimicrobial substances at their $\mathrm{MIC}_{50}$ values and to amoxicillin and ceftiofur also at their $\mathrm{MIC}_{90}$ value, both below the break-point of resistance. The exception was tetracycline where the majority of isolates were resistant (70\%), and the remaining $30 \%$ were only intermediately susceptible to tetracycline.

A higher level of resistance was detected to penicillin (20\%) in comparison with amoxicillin $(10 \%)$ and ceftiofur $(0 \%)$; resistance to enrofloxacin was $16.7 \%$ and to tulathromycin $13.3 \%$. The results of serotyping and MIC determination of 30 tested isolates against individual antimicrobials are summarized in Table 4. Nine isolates belonged to serotype 5 , three isolates were determined as serotype 13, and three isolates were serotype 14 . Two isolates were serotype 1 and other two isolates were serotype 12 and 4. A single isolate was identified as serotype 2 and another as serotype 15. Seven remaining isolates were serologically non-typable. The profiles of resistance of tested isolates are shown in Table 5.

Table 4. Minimum inhibitory concentration values $(\mathrm{mg} / \mathrm{l})$ of tested antimicrobials for Haemophilus parasuis isolates.

\begin{tabular}{|c|c|c|c|c|c|c|c|}
\hline Strain & Serotype & $\mathrm{PNC}$ & AMX & EFT & TUL & TET & ENR \\
\hline 1 & 15 & $\leq 0.06$ & $\leq 0.03$ & $\leq 0.125$ & 1 & 2 & $\leq 0.03$ \\
\hline 2 & NT & 0.25 & $\leq 0.03$ & $\leq 0.125$ & $\leq 0.5$ & 2 & $\leq 0.03$ \\
\hline 3 & 13 & 0.25 & 0.06 & 1 & $\leq 0.5$ & 2 & $\leq 0.03$ \\
\hline 4 & 2 & $\leq 0.06$ & $\leq 0.03$ & $\leq 0.125$ & 4 & 2 & 1 \\
\hline 5 & 1 & 0.25 & 0.06 & $\leq 0.125$ & 1 & 1 & $\leq 0.03$ \\
\hline 6 & 5 & 0.25 & 0.06 & $\leq 0.125$ & 1 & 1 & $\leq 0.03$ \\
\hline 7 & 1 & $\leq 0.06$ & $\leq 0.03$ & 0.25 & 2 & 2 & $\leq 0.03$ \\
\hline 8 & 4 & 0.25 & $\leq 0.03$ & 1 & 16 & 2 & $\leq 0.03$ \\
\hline 9 & NT & 0.25 & 0.06 & $\leq 0.125$ & 8 & 2 & $\leq 0.03$ \\
\hline 10 & NT & 2 & 0.25 & $\leq 0.125$ & 64 & 32 & 2 \\
\hline 11 & 13 & 0.5 & 0.125 & $\leq 0.125$ & 1 & 2 & $\leq 0.03$ \\
\hline 12 & 4 & 0.125 & 0.06 & 1 & $\leq 0.5$ & 2 & 0.25 \\
\hline 13 & NT & 2 & 0.25 & 0.25 & 64 & 64 & 1 \\
\hline 14 & NT & 8 & 4 & 0.5 & 16 & 64 & 2 \\
\hline 15 & 14 & 0.25 & 0.06 & $\leq 0.125$ & $\leq 0.5$ & 1 & $\leq 0.03$ \\
\hline 16 & 5 & $\leq 0.06$ & 0.06 & 0.25 & $\leq 0.5$ & 1 & $\leq 0.03$ \\
\hline 17 & 14 & 0.25 & 0.06 & $\leq 0.125$ & 2 & 2 & 0.125 \\
\hline 18 & 12 & 0.25 & 0.06 & 0.25 & 64 & 2 & 0.25 \\
\hline 19 & 5 & 0.25 & 0.06 & $\leq 0.125$ & $\leq 0.5$ & 2 & 0.06 \\
\hline 20 & 12 & 0.25 & 0.06 & 0.5 & $\leq 0.5$ & 1 & 0.125 \\
\hline 21 & 5 & 0.25 & $\leq 0.03$ & $\leq 0.125$ & 1 & 1 & $\leq 0.03$ \\
\hline 22 & NT & 4 & 0.25 & $\leq 0.125$ & 8 & 64 & 1 \\
\hline 23 & 5 & 8 & 4 & 0.5 & 32 & 64 & 0.125 \\
\hline 24 & 13 & 8 & 4 & $\leq 0.125$ & 4 & 2 & $\leq 0.03$ \\
\hline 25 & 5 & 0.125 & $\leq 0.03$ & 0.5 & 2 & 4 & $\leq 0.03$ \\
\hline 26 & 5 & $\leq 0.06$ & $\leq 0.03$ & 0.5 & 4 & 1 & $\leq 0.03$ \\
\hline 27 & 5 & 0.125 & $\leq 0.03$ & 0.5 & 1 & 1 & $\leq 0.03$ \\
\hline 28 & 5 & $\leq 0.06$ & $\leq 0.03$ & 0.25 & 1 & 2 & 0.06 \\
\hline 29 & 14 & 0.5 & 0.125 & $\leq 0.125$ & 2 & 1 & $\leq 0.03$ \\
\hline 30 & NT & 0.25 & 0.06 & $\leq 0.125$ & 64 & 2 & 0.125 \\
\hline
\end{tabular}

PNC - penicillin; AMX - amoxicillin; EFT - ceftiofur; TUL - tulathromycine; TET - tetracycline; ENR enrofloxacin; $\mathrm{PC}$ - positive growth control; NT - non-typable 
We found $3(10 \%)$ isolates resistant to three antimicrobials and isolates $(10 \%)$ resistant to four antimicrobials.

Table 5. The profiles of resistance of isolates Haemophilus parasuis $(\mathrm{n}=30)$.

\begin{tabular}{llcc}
\hline $\begin{array}{l}\text { Frequency } \\
\text { of resistance } \\
\text { by active } \\
\text { substance }\end{array}$ & $\begin{array}{l}\text { Phenotypic profile } \\
\text { of resistance }\end{array}$ & $\begin{array}{c}\text { Number } \\
\text { of isolates (\%) }\end{array}$ & $\begin{array}{c}\text { Number } \\
\text { of isolates (\%) } \\
\text { cumulatively } \\
\text { within groups }\end{array}$ \\
\hline $0^{*}$ & TET & $9(30.0)$ & $9(30.0)$ \\
1 & TET, ENR & $12(40.0)$ & $12(40.0)$ \\
2 & TET, TUL & $1(3.3)$ & $3(10.0)$ \\
2 & TET, PNC, AMX & $2(6.7)$ & $3(10.0)$ \\
3 & TET, PNC, ENR & $2(6.7)$ & \\
3 & TET, PNC, TUL, ENR & $1(3.3)$ & $3(10.0)$ \\
4 & TET, PNC, AMX, ENR & $2(6.7)$ & $1(3.3)$ \\
\hline
\end{tabular}

TET - tetracycline; ENR - enrofloxacin; TUL - tulathromycin; PNC - penicillin; AMX - amoxicillin *Isolates were susceptible to amoxicillin, penicillin, ceftiofur, enfofloxacin, tulathromycin, and intermediately susceptible to tetracycline.

\section{Discussion}

A total of 15 serovars have been recognised in $H$. parasuis till now and a large number of non-typable isolates have been described. Besides serovar 5, which is considered as the most prevalent and virulent one, serovars $1,2,4,8,10$, and 12-14 may lead to the death of infected animals and are considered virulent. Serovars 3, 6, 7, 9 and 11 are considered as avirulent(Kielstein and Rapp-Gabriels on 1992). The Czech isolates belong to serotypes $1,2,4,5,12,13,14$, and 15, with the most frequent serovars isolated among them belonging to the virulent group of 5,13, and 14 which are considered as highly virulent. Seven isolates $(23 \%)$ were non-typable, which is similar to Germany $(26 \%)$, but higher compared to Denmark and China (15\% and $12 \%$ of isolates, respectively) (Angen et al. 2004; Kielstein and Rapp-Gabrielson 1992; Cai et al. 2005).

Antimicrobial treatment remains the most important tool to control $H$. parasuis infection under real field conditions due to the lack of commercially available, effective and broadly protective vaccines as the inconsistency of their effect is due to serovar diversity and a high number of non-typable isolates (Oliveira and Pijoan 2004).

Antibiotic-wise, tetracycline was an exception, as all of the tested isolates were either resistant or showed an intermediate sensitivity pattern ( $70 \%$ and $30 \%$, respectively). High level of resistance to tetracycline is in agreement with findings in previous studies and our previously published results (Wissing et al. 2001; Pejsak et al. 2005; Dayao et al. 2014). In contrast, low level of resistance to tetracycline has been reported from China $(3.6 \%)$ but a different breakpoint $\leq 8 \mu \mathrm{g} / \mathrm{ml}$ was used in the study (Zhou et al. 2010).

Surprisingly, we have found $5(16.7 \%)$ isolates resistant to enrofloxacin with a bimodal distribution of MICs. This is the first evidence of enrofloxacin resistance reported in field isolates of $H$. parasuis in the Czech Republic. Fluoroquinolones are registered and used in the Czech Republic for the treatment of respiratory infections caused by Mycoplasma hyopneumoniae, Pasteurella multocida, Actinobacillus pleuropneumoniae as well as $H$. parasuis. Quinolone resistance typically develops in a stepwise manner and crossresistance between different members of the group is common (Chen et al. 2011). 
Similarly, strains with reduced susceptibility to fluoroquinolones have been described in Denmark (Aarestrup et al. 2004), and a high level of resistance was recently reported for enrofloxacin in China (70.9\%) (Zhou et al. 2010). Fluoroquinolone resistance could be associated with virulence factors, thus highly virulent strains are more strongly exposed to antibiotics in their evolutionary history, and proper selection of strains during MIC monitoring of such serotypes is very important (Zhang et al. 2013).

According to the current interpretative criteria for respiratory pathogens (CLSI 2013a; CLSI 2013b) the authors have found four (13.3\%) resistant isolates to tulathromycin. This finding is in accordance with our previous reported results from the Czech Republic (Nedbalcova etal.2013). Taking into consideration the pharmacokinetic data characteristic for tulathromycin - the low level plasma concentration $(0.62 \mu \mathrm{g}$ tulathromycin $/ \mathrm{ml})$, the rapid concentration of tulathromycin into the lungs and the septicaemic character/nature of the pathogen, tulathromycin may be less effective for treatment and control of $H$. parasuis infection (Benchaoui et al. 2004; Evans 2005; Ritzmann and Heinritzi 2005). MIC data demonstrating a sensitivity and resistance pattern for $H$. parasuis and tulathromycin suggest that an epidemiological cut-off value occurs at $4.0 \mu \mathrm{g} / \mathrm{ml}$ (Godino et al. 2005). Based on this proposed criteria, $12(40 \%)$ isolates from our study would belong to a resistance pattern. Tulathromycin resistance has recently been identified in $H$. parasuis isolates from Australia (Daya o et al. 2014). An experimental challenge study demonstrated a failure in protection and development of polyserositis with a high isolation rate of $H$. parasuis with the metaphylactic use of tulathromycin (Palzer at al. 2015).

A low degree of resistance was recorded to amoxicillin (3 strains $/ 10 \%$ ); all the rest of the tested isolates belong to a fully sensitive pattern, in contrast with another $\beta$-lactam antibiotic - penicillin, which has a comparatively higher degree of resistant isolates $(20 \%)$. The level of resistance to aminopenicillins remains relatively stable and low in the Czech Republic in comparison with data from Spain and the United Kingdom where a higher level of resistance was found to aminopenicillins $(66.7 \%$ and $21.1 \%$, respectively) (de la Fuente et al. 2007). Aminopenicillins are also frequently used in other countries with reported high resistance to tetracycline in clinical strains $H$. parasuis (San Millan et al. 2007). Aminopenicillins remain one of the most important antimicrobials for treatment and control of $H$. parasuis infections in the Czech Republic's field conditions. All the tested strains were sensitive to ceftiofur.

In conclusion, this study showed that the Czech isolates of $H$. parasuis are generally susceptible to amoxicillin and ceftiofur and these antimicrobial agents should be the preferred option used for the treatment of infection caused by $H$. parasuis. Recently, the impact of third generation cephalosporins, such as ceftiofur, in veterinary medicine has been discussed and has led to the emergence and dissemination of resistant organisms producing extended-spectrum beta-lactamases (ESBLs). Also, potential transmission from animals to humans mainly via the food chain has been widely postulated (Hammerum et al. 2014), so the use of ceftiofur should be considered as a last resort option.

\section{Acknowledgements}

This work was supported by the Ministry of Agriculture of the Czech Republic (Project No. RO 0516) and the Ministry of Education, Youth and Sports of the Czech Republic (Project No. LO 1218 NPU I).

\section{References}

Aarestrup F, Seyfarth A, Angen O 2004: Antimicrobial susceptibility of Haemophilus parasuis and Histophilus somni from pigs and cattle in Denmark. Vet Microbiol 101: 143-146

Angen O, Svensmark B, Mittal K 2004: Serological characterization of Danish Haemophilus parasuis isolates. Vet Microbiol 103: 255-258

Amano H, Shibata M, Kajio N, Morozumi T 1994: Pathologic observations of pigs intranasally inoculated with serovar 1, 4 and 5 of Haemophilus parasuis using immunoperoxidase method. J Vet Med Sci 56: 639-644 
Benchaoui HA, Nowakowski M, Sherington J, Rowan TG, Sunderland SJ 2004: Pharmacokinetics and lung concentrations of tulathromycin in swine. J Vet Pharmacol Ther 27: 203-210

Cai X, Chen H, Blackall PJ, Yin Z, Wang L, Liu Z, Jin M 2005: Serological characterization of Haemophilus parasuis isolates from China. Vet Microbiol 111: 231-236

CLSI 2013a: Performance Standards for Antimicrobial Disk and Dilution Susceptibility Tests for Bacteria Isolated from Animals; Approved Standard $-4^{\text {th }}$ edn. CLSI document Vet 01-A4. Clinical and Laboratory Standards Institute, Wayne, PA 2013

CLSI 2013b: Performance Standards for Antimicrobial Disk and Dilution Susceptibility Tests for Bacteria Isolated from Animals; Second Informational Supplement. CLSI document Vet 01-S2. Clinical and Laboratory Standards Institute, Wayne, PA 2013

Dayao DAE, Kienzle M, Gibson JS 2014: Use of a proposed antimicrobial susceptibility testing method for Haemophilus parasuis. Vet Microbiol 172: 586-589

De la Funete AJ, Tucker AW, Navas J, Blanco M, Morfia SJ, Gutiérrez-Martín CB 2007 Antimicrobial susceptibility patterns of Haemophilus parasuis from pigs in the United Kingdom and Spain. Vet Microbiol 120: 184-191

Godinho K, Keane S, Nanjiani I, Benchaoui H, Sunderland S, Jones M, Weatherley A, Gootz T, Rowan T 2005: Minimum inhibitory concentrations of tulathromycin against respiratory bacterial pathogens isolated from clinical cases in European cattle and swine and variability arising from changes in vitro methodology. Vet Ther 6: 113-121

Evans NA 2005: Tulathromycin: an overview of a new triamilide antibiotic for livestock respiratory disease. Vet Ther 6: 83-95

Hammerum AL, Larsen J, Andersen VD, Lester CH, Skovgaard Skytte TS, Hansen F, Olsen SS, Mordhorst H, Skov RL, Aarestrup FM, Agersø Y 2014: Characterization of extended-spectrum $\beta$-lactamase (ESBL)producing Escherichia coli obtained from Danish pigs, pig farmers and their families from farms with high or no consumption of third- or fourth-generation cephalosporins. J Antimicrob Chemother 69: 2650-2657

Chen P, Liu Y, Liu Ch, Zou H, Wang Y, Li W, Bi D, He Q 2011: Laboratory detection of Haemophilus parasuis with decreased susceptibility to nalidixic acid and enrofloxacin due to GyrA and ParC mutations. J Anim Vet Adv 10: 2870-2873

Kielstein P, Rapp-Gabrielson VJ 1992: Designation of 15 serovars of Haemophilus parasuis based immunodifusion using heatstable antigen extracts. J Clin Microbiol 30: 862-865

Mittal KR, Higgins R, Larivière S 1983: Identification and serotyping of Haemophilus pleuropneumoniae by coagglutination test. J Clin Microbiol 18: 1351-1354

Nedbalcova K, Kucerova Z 2013: Antimicrobial susceptibility of Pasteurella multocida and Haemophilus parasuis isolates associated with porcine pneumonia. Acta Vet Brno 82: 3-7

Nicolet J 1992: Haemophilus parasuis. In: Diseases of swine. $7^{\text {th }}$ edn; Iowa State University Press, 1992: 526-528

Oliveira S, Galina L, Pijoan C 2001: Development of a PCR test to diagnose Haemophilus parasuis infections. J Vet Diagn Invest 13: 495-501

Oliveira S, Pijoan C 2004: Haemophilus parasuis: new trends on diagnosis, epidemiology and control. Vet Microbiol 99: 1-12

Palzer A, Austin-Busse RL, Ladinig A, Gyula Balka G, Spergser J, Ritzmann 2015: Different sample types in pigs challenged with Haemophilus parasuis following two treatment schemes with tulathromycin. A Vet Hungarica 63: $157-166$

Pejsak Z, Jabonski A, Zmudzki J, 2005: Drug sensitivity of pathogenic bacteria isolated from the respiratory system of swine. Med Wet 61: 664-668

Ritzmann M, Heinritzi K 2005: Klinisches Bild, Diagnostik und Differenzialdiagnostik der Glässerschen Krankheit. Tierarztl Prax 61-64

San Millan A, Escudero J, Catalan A, Nieto S, Farelo F, Gibert M, Moreno M, Dominguez L, Gonzalez-Zorn B 2007: Beta-lactam resistance in Haemophilus parasuis is mediated by plasmid pB1000 bearing blaROB-1. Antimicrob Agents Chemother 51: 2260-2264

Schwarz S, Chaslus-Dancla E 2001: Use of antimicrobials in veterinary medicine and mechanisms of resistance. Vet Res 32: 201-225

Schwarz S, Silley P, Simjee S, Woodford N, van Duijkeren E, Johnson AP, Gaastra W 2010: Assessing the antimicrobial susceptibility of bacteria obtained from animals. Vet Microbiol 141: 1-4

Wissing A, Nicolet J, Berlin P 2001: Antimicrobial resistance situation in Swiss veterinary medicine. Schweiz Arch Tierheilkd 143: 503-510

Zhang Q, Liu J, Yan S, Yang Y, Zhang A, Jin M 2013: Fluoroquinolone-resistant Haemophilus parasuis isolates exhibit more putative virulence factors than their susceptible counterparts. J Clin Microbiol 51: 3130-3131

Zhou X, Xu X, Zhao Y, Chen P, Zhang X, Chen H, Cai X 2010: Distribution of antimicrobial resistance among different serovars of Haemophilus parasuis isolates. Vet Microbiol 141: 168-173 